\title{
Behavioral And Physiological Analysis In A Zebrafish Model Of Epilepsy
}

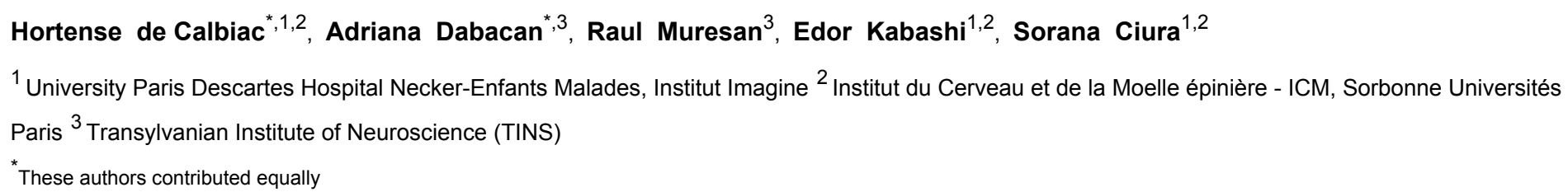

\section{Corresponding Author}

\section{Sorana Ciura}

sorana.ciura@institutimagine.org

\section{Citation}

de Calbiac, H., Dabacan, A.,

Muresan, R., Kabashi, E.,

Ciura, S. Behavioral And Physiological

Analysis In A Zebrafish Model Of

Epilepsy. J. Vis. Exp. (176), e58837,

doi:10.3791/58837 (2021).

\section{Date Published}

October 19, 2021

DOI

$10.3791 / 58837$

URL

jove.com/video/58837

\section{Abstract}

Epilepsy represents one of the most common neurological disorders, affecting an estimated 50 million people worldwide. Recent advances in genetic research have uncovered a large spectrum of genes implicated in various forms of epilepsy, highlighting the heterogeneous nature of this disorder. Appropriate animal models are essential for investigating the pathological mechanisms triggered by genetic mutations implicated in epilepsy and for developing specialized, targeted therapies. In recent years, zebrafish has emerged as a valuable vertebrate organism for modeling epilepsies, with the use of both genetic manipulation and exposure to known epileptogenic drugs, such as pentylenetetrazole (PTZ), to identify novel antiepileptic therapeutics. Deleterious mutations in the mTOR regulator DEPDC5 have been associated with various forms of focal epilepsies and knock-down of the zebrafish orthologue causes hyperactivity associated with spontaneous seizure-like episodes, as well as enhanced electrographic activity and characteristic turn wheel swimming. Here, we described the method involved in generating the DEPDC5 loss-of-function model and illustrate the protocol for assessing motor activity at 28 and $48 \mathrm{~h}$ post fertilization (hpf), as well as a method for recording field activity in the zebrafish optic tectum. An illustration of the effect of the epileptogenic drug PTZ on neuronal activity over time is also provided.

\section{Introduction}

Due to its small size, oviparous development and transparency at early stages of development, zebrafish has emerged as a valuable vertebrate organism for modeling human diseases as diverse as cardiovascular, cancer or neurological disorders ${ }^{1,2}$. Zebrafish combines the advantages of a vertebrate, including the high conservation of organ architecture and genetic code, with the small size and ease of genetic manipulation of simpler model organisms, therefore facilitating both fundamental studies and translational applications. In particular, its amenability 
to high-throughput automated screening of behavior and fluorescent markers of cellular processes has made zebrafish a particularly attractive model for epilepsy research. This has been demonstrated by a high increase in the last decade of the number of publications featuring chemically-induced and/or genetic models of epilepsy $3,4,5$ and, more recently, reports of promising therapeutics obtained from chemical screens in these models ${ }^{6,7,8}$.

DEPDC5 is a member of the GATOR1 complex, a negative regulator of mTOR signaling9. Mutations in the DEPDC5 gene have been first discovered in 2013 in probands suffering from autosomal dominant focal epilepsies ${ }^{10,11}$, and have since been reported in a number of clinical conditions associated with focal epileptic manifestations and focal cortical dysplasia ${ }^{12}$. The large majority of reported mutations are predicted to cause the loss-of-function of the gene ${ }^{12}$, and this was formally demonstrated for a number of DEPDC5 mutated transcripts which are targeted by nonsense mediated mRNA decay ${ }^{12,13}$. In agreement, knock-down of the gene orthologue in zebrafish using antisense morpholino oligonucleotides (AMOs) results in a number of features that are common to epileptic models in this organism, including hyperactivity, turn wheel-like swimming, spontaneous seizures and enhanced neuronal activity $14,15,16,17,18$. Interestingly, treatment with rapamycin, an inhibitor of mTOR signaling, reversed the behavioral features of this model ${ }^{18}$, supporting the hypothesis that $D E P D C 5$ loss-of-function can trigger epilepsy due to a misregulation of the mTOR pathway ${ }^{9}, 19$.

Transient knock-down of gene expression in vivo using antisense oligonucleotides carrying the morpholino modification has been an invaluable tool for studying the role of specific genes, on par with si/shRNA-based techniques. Recently, AMO-based strategies have also found clinical applications, with a first AMO therapy receiving the FDA approval for the treatment of Duchenne muscular atrophy in $2016^{20}$. While it was reported that in zebrafish the phenotype of acute AMO-based gene knock-down does not always correlate with the constitutive knock-out models ${ }^{21}$, this can be due at least in some instances to compensatory mechanisms engendered by constitutive genetic modifications ${ }^{22}$. However, the issue of specificity of the AMO-induced phenotype is an undisputable concern that has to be diligently addressed in studies using this technology ${ }^{23}$. In order to ensure the specificity of the AMObased knock-down phenotype, several key controls are necessary. These include a dose-response curve that allows the selection of the lowest dose of AMO effective for gene knock-down, avoiding overall toxicity due to the introduction of an excess of genetic material. The use of a Mismatch AMO that does not target any particular region in the genome is also required for establishing an appropriate dose and in identifying a specific phenotype. A second AMO which targets a different region of the same gene, such as a spliceblocking $\mathrm{AMO}$, is necessary to confirm that the phenotype is due to the knock-down of the target gene. Rescue of the knock-down phenotype with the cDNA of the gene, either the human orthologue or a codon-modified version of the zebrafish gene that cannot be targeted by the AMO, provides a strong argument in favor of the phenotype specificity. Lack of rescue with the same cDNA containing loss-of-function mutations (such as the introduction of an early stop codons) is a further proof in this direction.

Here, we present a method for generating a zebrafish $D E P D C 5$ loss-of-function model and the protocol for behavioral phenotyping at 28 and $48 \mathrm{~h}$ post fertilization (hpf). At $28 \mathrm{hpf}, D E P D C 5$ loss-of-function causes overall 
hyperactivity, as evidenced by enhanced coiling and twitching movements of the embryos within the chorion. An automated motion detection system can be used at this stage to quantify the overall activity per embryo. At $48 \mathrm{hpf}$, zebrafish exhibit stereotyped escape swimming in response to touch. In zebrafish with downregulated expression of DEPDC5, the swimming trajectory is significantly more tortuous than in controls, the fish exhibiting a "cork-screw" or "turn-wheel" like pattern, similar to other reported epilepsy models in this organism ${ }^{3,4}$. Electrophysiological recordings were obtained in the optic tectum in zebrafish larvae between 4-6 days post fertilization (dpf) and show a baseline increase in neuronal activity in the DEPDC5 knock-down animals. The advantage of this model is that it presents several phenotypic features at different time points, which can be useful in monitoring and assessing the efficacy of drug therapies during development.

\section{Protocol}

Experimental procedures were approved by the National and Institutional Ethical Committees.

\section{Transient Knock-down of DEPDC5 Gene in Zebrafish Embryo}

\section{Preparation of tools:}

1. Prepare silicon elastomer-coated injection Petri dishes: Mix the base and curing agent of the kit (see Table of Materials) in a 10:1 ratio. Fill a $35 \mathrm{~mm}$ Petri dish halfway with the mixture. Wait for the silicon to harden before using it (this can take several days).

2. Prepare $1.2 \mathrm{mmol} / \mathrm{L}$ stock solutions of antisense morpholino oligonucleotides (AMO; see Table of Materials). Add $250 \mu \mathrm{L}$ of sterile water to 300 nmole lyophilized AMO to obtain a $1.2 \mathrm{mmol} / \mathrm{L}$ stock solution. For complete dissolution, heat the vials for
5 min at $65^{\circ} \mathrm{C}$. Vortex briefly. Seal the tube cap with a plastic film (see Table of Materials).

3. For the control rescue experiments, prepare an expression plasmid containing the human CDNA of DEPDC5 (see Table of Materials) cloned in the pCS2 backbone or a similar zebrafish-compatible expression plasmid. As a negative control, a mutation causing an early stop codon (p.Arg487*) was introduced in the cDNA.

4. Prepare embryo water: $0.06 \mathrm{~g} / \mathrm{L}$ aquarium salt (see Table of Materials) in reverse osmosis water +0.5 $\mathrm{mg} / \mathrm{L}$ methylene blue.

5. The day of the injection, prepare microinjection borosilicate glass needles using a puller (see Table of Materials). Establish appropriate temperature settings on the needle puller. Use a $10 \mathrm{~cm}$ long, 1/0.5 OD/ID mm borosilicate glass capillary to generate two $\sim 5 \mathrm{~cm}$ capillaries with thin tips with a length of approximately $1 \mathrm{~cm}$.

6. If the tip of the needles is very fine, preventing the ejection of solution, break the very end of the tapered tip using forceps under a microscope.

7. Just prior to injection, prepare the working solutions of AMOs. Always prepare fresh solution to ensure the reproducibility of the results. Heat the AMO stock vials at $65{ }^{\circ} \mathrm{C}$ for $5 \mathrm{~min}$. Prepare a $5 \mu \mathrm{L}$ injection sample containing Fast Green dye $(0.02 \%$ final concentration, see Table of Materials) and the AMO diluted at the working concentration in water.

8. Determine the working concentration of the AMO empirically for each gene using a dose response curve. The working concentration represents a concentration where the AMO is effective in 
knocking down the gene without causing general toxicity, such as gross morphological defects. Typically, AMO working concentrations will be in a range of $0.2 \mathrm{mmol} / \mathrm{L}$ to $1 \mathrm{mmol} / \mathrm{L}(0.4 \mathrm{mmol} / \mathrm{L}$ was determined as the effective concentration for this study ${ }^{18}$ ). Inject the control Mismatch morpholino at the same concentration as the effective AMO.

9. Vortex the tubes and centrifuge briefly to bring the droplets to the bottom of the tubes.

10. For rescue experiments, prepare a $5 \mu \mathrm{L}$ injection sample with the AMO diluted at the working concentration and the cDNA expression plasmid diluted in water to a final concentration to be determined empirically. For the expression of $D E P D C 5$ and the negative control plasmid, $100 \mathrm{ng} /$ $\mu \mathrm{L}$ was effective for phenotypic rescue.

\section{Embryo preparation:}

1. The day prior to microinjection, set up the zebrafish mating tanks. The morning of the injection, remove the dividers to enable spawning. Collect the eggs in $100 \mathrm{~mm}$ Petri dishes filled with embryo water using a fine sieve. Inject within 20-30 min from collection, while the eggs are at the one cell stage.

2. Pick $60-80$ eggs with a plastic Pasteur pipette and arrange them in the silicon-coated Petri dish for injection. The silicon surface will prevent the eggs from sliding during the injections. Remove most of the embryo water, leaving just enough to cover the eggs halfway.

\section{Microinjections:}

1. Fill a glass needle with injection solution. Place the needle vertically in one of the tubes containing the injection solution, ensuring that the bottom end of the needle is touching the solution. Wait several minutes until the colored injection solution rises by capillarity and is visible at the tip of the needle.

2. Mount the filled needle on the injecting handle of the microinjector (see Table of Materials).

3. Turn on the air compressor and adjust the pressure setting to generate an injection volume of $\sim 2 \mathrm{~nL}$.

4. To calculate the volume of the injected solution, place a drop of mineral oil on a microtome slide. Inject the dye-containing solution using the set pressure and time parameters. Measure the diameter of the injected fluid sphere and calculate total volume using the formula Volume $=4 / 3^{*} \pi^{*}(d / 2)^{3}$, where $d=$ the measured diameter of the injected bolus.

5. Using a dissecting binocular microscope with a $4 X$ magnification, inject the eggs at the single cell stage by passing through the chorion and the yolk, and projecting the solution directly within the cell.

6. Collect the injected embryos in a $100 \mathrm{~mm}$ Petri dish with embryo water, label the dish and incubate them at $28^{\circ} \mathrm{C}$.

7. Ensure that the incubator temperature is stable over time, as embryos development rate is thermosensitive. For example, the growth would be accelerated at higher temperatures and the developmental stage is critical for properly assessing the phenotype ${ }^{24}$.

8. Check the quality of the eggs 6-8 $\mathrm{h}$ after injection and remove dead and unfertilized embryos using a plastic Pasteur pipette. 
9. The next morning, count and remove dead embryos in each dish with a plastic Pasteur pipette.

\section{Behavior Analysis}

\section{Global activity analysis at $\mathbf{2 8} \mathrm{hpf}$ :}

1. Conduct the test the afternoon of the day after microinjection (28 hpf), ensuring that the time of day at which the test is conducted is consistent over experiments to perform valid statistical analysis as embryos development is very rapid.

2. Fill a $35 \mathrm{~mm}$ dish (test dish) with embryo water and allow it to warm up in the incubator $\left(28^{\circ} \mathrm{C}\right)$ for at least 15 min prior to starting the test.

3. Place a plastic mesh grid $(1.2 \times 1.2 \mathrm{~mm})$ cut to size, on the bottom of the test dish.

4. Have another experimenter randomize the order of testing of the embryos and mask the names of the conditions to be tested.

5. Make sure that the mortality rate does not change among conditions and compared to non-injected embryos to ensure the specificity of the phenotype. The percent of dead embryos in all conditions shall not exceed $10-13 \%^{18}$.

6. Place $10-12$ embryos still within their chorion on the plastic mesh using a plastic Pasteur pipette. Fill the test dish with enough embryo water to keep the embryos submerged but not floating. If needed, move the embryos with care using a plastic tip to position them on the grid.

7. Using a video camera (see Table of Materials) attached to a dissection microscope, record the spontaneous coiling activity for a defined length of time (10-20 min long videos are usually sufficient to obtain representative samples of activity bursts for the quantification)

8. Return the embryos to their respective dish and put them back in the incubator. Repeat the experiment with as many embryos as needed for each condition (as determined by a $90 \%$ power analysis).

9. To analyze total spontaneous movement, use a ZebraLab system (see Table of Materials). Using the activity quantification module, upload the recorded video and design the tracking arenas around each embryo as appropriate. Set the freeze and burst threshold to 10 and 50, respectively.

10. Run the automated video analysis, which quantifies total activity inside each of the defined arenas, then recover the data set as a spreadsheet and perform the analysis using a data analysis software.

\section{Touch-Evoked-Escape-Response (TEER) at $48 \mathrm{hpf:}$}

1. Conduct the test in the morning two days after the injection (48 $\mathrm{h}$ after the fertilization).

2. At least $2 \mathrm{~h}$ prior to testing, dechorionate the embryos using fine forceps. Ensure that the time of day for the dechorionation and the behavior test is consistent over experiments.

3. Fill a $130 \mathrm{~mm}$ dish (test dish) with embryo water and allow it to warm up in the incubator $\left(28^{\circ} \mathrm{C}\right)$ for at least 15 min prior to starting the test.

4. Count and remove dead and morphologically deformed larvae. Record the numbers for each condition.

5. Have another experimenter randomize the order and codify the names of the conditions to be tested. 
6. Mount the camera (see Table of Materials) over the test dish making sure that the entire test dish is within the field of view. Placing a ruler within the field of view provides an internal calibration for distance.

7. With a plastic Pasteur pipette, put an embryo in the center of the test dish and begin the recording using an acquisition rate of $30 \mathrm{fps}$.

8. With a fine plastic tip, touch slightly the tail of the embryo with a flicking motion.

9. Stop the recording when the larva has terminated its movement.

10. Remove the embryo from the test dish and place it in a new dish filled with embryo water. Repeat the test with as many embryos as needed for each condition (as determined by a $90 \%$ power analysis).

11. Return the embryos to their original dish and put them back in the incubator.

12. To analyze the parameters of the swimming behavior, load the recorded video to Image J analysis software. Download and install the Manual Tracking plugin of ImageJ (see Table of Materials). Launch the plugin by choosing Tools | Plugin | Manual Tracking in the menu.

13. In the dialog window, introduce the calibrated scale of the image. Including a ruler in the camera field facilitates the conversion of $\mathrm{cm}$ to pixels.

14. Select Add track and start the trajectory tracing by clicking on the image of the zebrafish larva in the first frame. The frames advance automatically with each point that is added to the trace.

15. Continue tracing the movement until the end of the swimming episode.
16. Select End track on tracking window, retrieve the $X$ $Y$ coordinates and calculate total distance, velocity and turning angle.

\section{Electrophysiological Analysis}

1. Reagent and tool preparation:

1. Prepare $1 \%$ agarose in embryo water (see section 1.1.4). Aliquot the liquid agarose in microcentrifuge tubes and keep these on a heating block at $42^{\circ} \mathrm{C}$ to prevent the agarose from hardening.

2. Prepare the recording solution (in $\mathrm{mmol} / \mathrm{L}$ ): $\mathrm{NaCl}$ 134, $\mathrm{KCl} 2.9, \mathrm{CaCl} 2$ 2.1, $\mathrm{MgCl} 2$ 1.2, glucose 10, HEPES 10, pH 7.8.

3. Pull borosilicate glass micropipettes with a tip opening of $1.5-2 \quad \mu \mathrm{m} \quad(5-6$ $\mathrm{Mm}^{2} \cdot \mathrm{kg} \cdot \mathrm{s}^{-3} \cdot \mathrm{A}^{-2}$ resistance) unpolished.

2. Preparation of zebrafish larvae for electrophysiology:

1. Place the fish in a glass-bottom Petri dish (see Table of Materials) and remove excess extracellular media in order to ensure the fish is brought as close to the cover slip as possible.

2. Using a plastic Pasteur pipette, add warm liquid agarose on and around the larva. Use just enough agarose to cover the fish. While the agarose hardens, use fine forceps to orient the fish in a straight position, ventral side down, in the center of the dish.

3. Add $2 \mathrm{~mL}$ of the recording solution containing $10 \mu \mathrm{M}$ Pancuronium bromide (see Table of Materials) to block neuromuscular transmission. The addition of 
the paralyzer is necessary to eliminate artefacts due to small movements during the recordings.

\section{Electrophysiological recording}

1. Fill the micropipette with recording solution.

2. With the patch clamp amplifier (see Table of Materials) in voltage clamp configuration, measure electrode resistance in bath to confirm its correct value.

3. Using a 20x objective, position the head of the larva in the central field of view and lower the micropipette to reach the recording position in the brain, within the optic tectum.

4. Switch the patch clamp amplifier to current clamp and fix the holding current to $0 \mathrm{~mA}$.

5. Using a low-pass filter of $1 \mathrm{kHz}$, an acquisition rate of $1 \mathrm{kHz}$ and a digital gain of 10 , record spontaneous activity for $60 \mathrm{~min}$ for determining baseline activity levels.

6. After $1 \mathrm{~h}$ of baseline recording, add $200 \mu \mathrm{L}$ pentylenetetrazol (PTZ, see Table of Materials) solution $300 \mathrm{mmol} / \mathrm{L}$ to the bath for a final concentration of $20 \mathrm{mmol} / \mathrm{L} \mathrm{PTZ}$.

7. Record the neuronal activity in PTZ for another 120 $\min$.

\section{Depolarization event determination}

1. Field recording events have very slow dynamics (frequencies of interest are in the range of 0.005-0.2 $\mathrm{s}^{-1}$ ). Therefore, filter the signal with a low pass (Butterworth 5th order LPF at $100 \mathrm{~s}^{-1}$ ) in order to avoid aliasing. Subsample the recorded voltage data from the acquisition frame rate (in this case, $1 \mathrm{ks}^{-1}$ ) down to $250 \mathrm{~s}^{-1}$ (RAW SIGNAL).

2. To identify the timestamps for each depolarization event, use a DETECTION SIGNAL, which is a high-pass filtered version of the recorded signal (Butterworth 1st order HPF at $0.01 \mathrm{~s}^{-1}$ ).

3. By eliminating the low frequency components, the detection of depolarization events can be performed using a simple thresholding method. Use a fixed threshold for noise elimination and event detection (0.3 mV was used for this study).

4. Characterize the depolarization event by a series of thresholds crossings that occur at time intervals smaller than $4 \mathrm{~s}$. Compute the start and the end of depolarization event as determined from consecutive sequences of threshold crossings. Events that are shorter than 40 ms can be discarded as noise.

5. Compute the amplitude of the events in the unfiltered (RAW SIGNAL) to eliminate errors due to the effect of the low pass filtering on the peak of the event. Select the depolarization wavelet from the raw signal using the timestamps determined in the filtered signal. Measure the amplitude as the difference between the maximum and minimum values of the wavelet selected from the raw signal.

NOTE: The script files to perform step $3.4-$ Depolarization event determination - and to obtain Figure 1 are provided as Supplementary File attached to this article. 


\section{Representative Results}

Figure 1 shows representative voltage traces of 4-6 dpf zebrafish larva extracellular field recordings in the case of two genetic conditions: Mismatch control and DEPDC5 knockdown. In the baseline period of the recording, DEPDC5 knockdown shows a higher occurrence of spontaneous events, while the Mismatch control displays very few fluctuations. These activity patterns are representative of the significant increase in neuronal activity due to loss-of-function of
$D E P D C 5$, as we have previously reported ${ }^{18}$. After PTZ application, both Mismatch control and DEPDC5 knock-down show an increased number of depolarization events. During the first period after PTZ application (10 - $60 \mathrm{~min})$, a rate of 0.8 events per min is observed in both Mismatch control and $D E P D C 5$ knock-down, where the majority of events are of high amplitude $(>1 \mathrm{mV})$. During the latter response period (60 - 120 min after PTZ application), the rate of depolarization events increases to around 1 event per min, and the majority of the events are of low amplitude ( $\leq 1 \mathrm{mV})$.

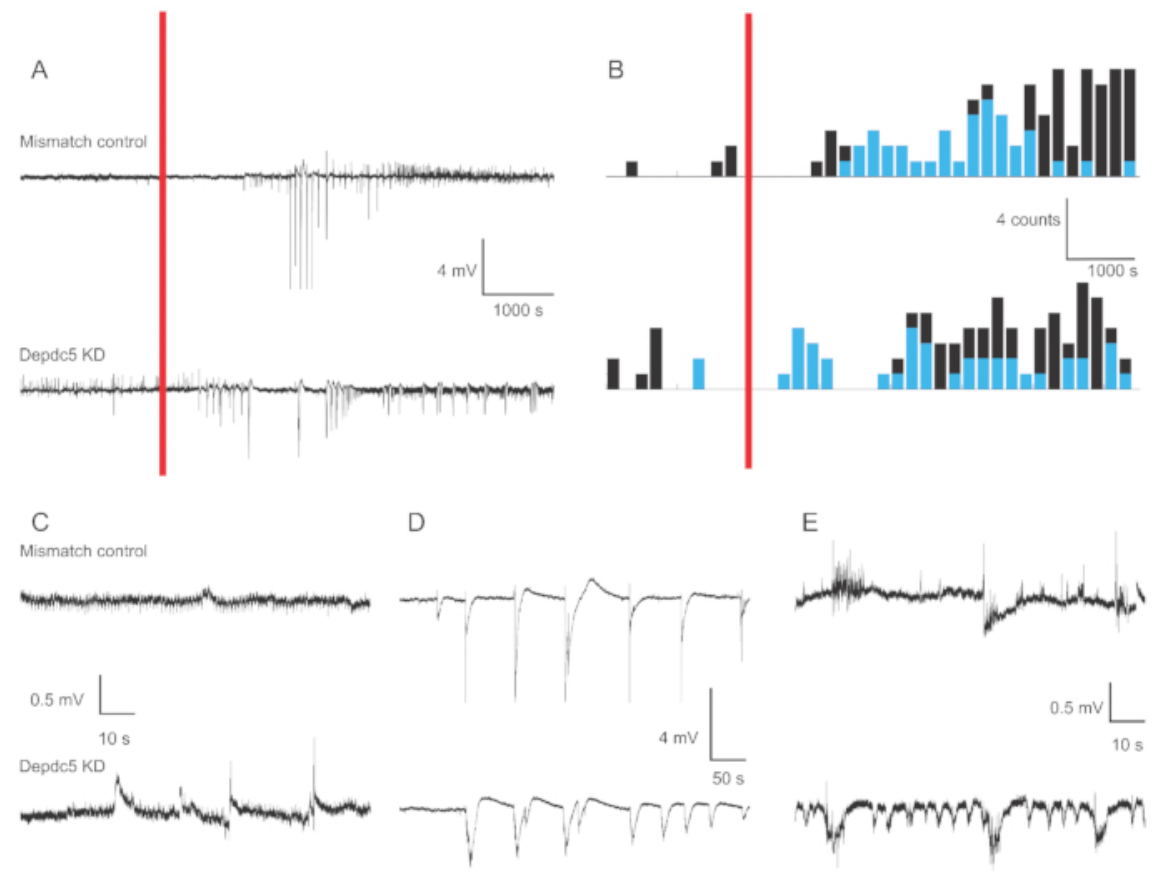

Figure 1: Example traces of field recordings in the zebrafish larvae brain. (A) Overview of 180 min recording for a Mismatch control larva and a DEPDC5 Knock-down. First, spontaneous baseline activity was recorded, then PTZ was applied in bath (red bar). (B) Peri-stimulus time histograms of the depolarization events for Mismatch control and DEPDC5 knock-down. The events were classified as high amplitude (>1 mV - blue) and low amplitude ( $\leq 1 \mathrm{mV}$ - black). (C-E) Example traces of the different periods of the recording: (C) spontaneous activity, (D) High amplitude events during the first period after PTZ application, (E) Low amplitude events during the latter period after PTZ application. Note that the script files to obtain these figures are provided as Supplementary File. Please click here to view a larger version of this figure. 
Supplementary File: Script files for step 3.4. Please click here to view a larger version of this figure.

\section{Discussion}

Epilepsy is a complex neurological disease, featuring a wide range of etiologies that are starting to be elucidated with the advent of genetic sequencing technologies $25,26,27$. Versatile animal models are essential for an efficient translational strategy that will yield both insights into the pathological mechanisms of genetically linked epilepsies, as well as targeted therapies for the distinct forms of this condition. Zebrafish models have been very effective at reproducing major features of epilepsy and providing reliable readouts for anti-epileptic drug screening ${ }^{5}, 28$. Spontaneous seizures can be detected in genetically modified zebrafish ${ }^{15,29,30,31}$ and neurophysiological analysis in these models ${ }^{28}$ has confirmed the neuronal basis of the epileptic-like behavior ${ }^{32,33}$. Smallsized zebrafish larvae are amenable to chemical screens in 96-well format using automated detection of simple behavior, such as spontaneous swimming, which allows for rapid detection of potential therapeutics.

The DEPDC5 knock-down model presented here is obtained by injection of AMO into the zebrafish embryo to block gene expression during development. This model presents several keystone phenotypic features during different time points of larval development, which can be used as indicators of therapy efficiency during a chemical or genetic screening protocol. The AMO-mediated gene knock-down is a powerful technique, displaying advantages over chemically-induced seizure models, as it specifically targets the expression of a gene of interest, thus allowing the identification of the underlying pathogenic mechanisms triggered by a genetic mutation. Chemical inducers, which are nevertheless potent tools for drug screenings, can act through multiple cellular pathways that might not be always relevant to the genetic mutation under study. While AMO injection is in itself a simple technique when mastered by the experimenter, it also presents a number of limitations. The injections have to be performed at the one cell stage embryo; in our hands, injections at later stages greatly increased the variability of the phenotype. This limits the time available for injection; therefore, a strategy of generating eggs for injection in a time sequence is useful. We routinely use $4-5$ crosses that we open at $15-20$ min intervals, allowing the injection of one clutch before obtaining the next one. Further, care must be taken to assess the phenotype at the same time points between different experiments, as stereotyped behaviors evolve rapidly during the first days of development. The volume and concentration of AMOs must also be carefully controlled, as general toxicity due to injecting excessive amounts will mask the specific phenotype. The different controls presented in the introduction are essential for determining the right injection dose and the corresponding phenotype.

Field recordings of the larval zebrafish brain are a useful tool for investigating the deleterious effects of genetic mutations involved in different brain disorders on the global neuronal activity ${ }^{34}$. Depolarization events seen under these experimental conditions are an established method for assessing electrophysiological effects of drugs in different epileptic conditions ${ }^{15,35}$. However, the assessment of these effects has mostly been done qualitatively rather than quantitatively, and having a subjective observer as an actor in the analysis. Here, we develop an automatic detection strategy that can objectively quantify the rate of depolarizations, their amplitude and duration, and can 
evaluate the progress of these parameters across time, or with different genetic or pharmacologic interventions.

The representative results presented here show the expected field activity of the DEPDC5 knock-down genetic model in comparison to a Mismatch control in 4-6 dpf zebrafish, before and after the application of PTZ to introduce epileptiformlike electrographic activity. Previously, we have shown a significant increase in the basal activity of the DEPDC5 knockdown condition $^{18}$. Here, we show that the response of these two conditions to PTZ, a chemical epileptiform activity inducer, has a similar trajectory in time, starting with a period of relatively low frequency, high amplitude depolarization events and continuing with a period of higher frequency, lower amplitude depolarization events. Field recording events have slow dynamics (frequencies of interest are in the range of 0.005-0.2 $\mathrm{s}^{-1}$ ), therefore both low-pass and high-pass filters are used in this protocol to isolate the events of interest. After eliminating the low frequency noise, the detection of depolarization events is performed using a simple threshold. Since the statistics of the signal are greatly affected by the presence of depolarization events, we could not use the standard deviation of the total signal to determine this threshold. The variability of the value of the standard deviation across datasets was greater than the observed recording noise levels. Therefore, after visual inspection of the traces, we used a fixed value of the threshold of $0.3 \mathrm{mV}$, in order to avoid the biasing induced by different levels of depolarization activity.

The described protocol provides a standardized and simple method for evaluating the motor behavior and the neuronal field activity, via extracellular current clamp voltage recording coupled with automatic detection of depolarization events in the optic tectum, to characterize epileptiform-like phenotypes in zebrafish models.

\section{Disclosures}

The authors have nothing to disclose.

\section{Acknowledgments}

We would like to thank the staff of the ICM electrophysiology platform where the neurophysiology experiments were performed. We also thank Anca Marian for technical help. SC was supported by the Trampoline Grant \#21488. EK was supported by the AFM Grant \#18469 and ERC Consolidator Grant (ALS-Networks). HC was supported by $\mathrm{PhD}$ awards from the Fondation pour la Recherche Médicale (PLP20141031462) and ARSLA. For AD and RM, this work was supported by three grants from the Romanian National Authority for Scientific Research and Innovation, CNCS-UEFISCDI (project numbers PN-IIIP4-ID-PCE-2016-0010, PN-III-P2-2.1-PED-2016-0007, and COFUND-NEURON-NMDAR-PSY), a grant by the European Union's Horizon 2020 research and innovation programme - grant agreement no. 668863-SyBil-AA, and a National Science Foundation grant NSF-IOS-1656830 funded by the US Government.

\section{References}

1. Kabashi, E., Champagne, N., Brustein, E., Drapeau, P. In the swim of things: Recent insights to neurogenetic disorders from zebrafish. Trends in Genetics. 26 (8), 373-381 (2010).

2. Baxendale, S., van Eeden, F., Wilkinson, R. The Power of Zebrafish in personalised medicine. Advances in Experimental Medicine and Biology. (2017). 
3. Baraban, S.C., Taylor, M.R., Castro, P.A., Baier, H. Pentylenetetrazole induced changes in zebrafish behavior, neural activity and c-fos expression. Neuroscience. (2005).

4. Cunliffe, V.T. Building a zebrafish toolkit for investigating the pathobiology of epilepsy and identifying new treatments for epileptic seizures. Journal of Neuroscience Methods. (2016).

5. Griffin, A., Krasniak, C., Baraban, S.C. Advancing epilepsy treatment through personalized genetic zebrafish models. Progress in Brain Research. (2016).

6. Griffin, A., Hamling, K.R., Knupp, K., Hong, S.G., Lee, L.P., Baraban, S.C. Clemizole and modulators of serotonin signalling suppress seizures in Dravet syndrome. Brain. (2017).

7. Orellana-Paucar, A.M. et al. Insights from zebrafish and mouse models on the activity and safety of arturmerone as a potential drug candidate for the treatment of epilepsy. PLoS ONE. (2013).

8. Baxendale, S. et al. Identification of compounds with anticonvulsant properties in a zebrafish model of epileptic seizures. Disease Models \& Mechanisms. (2012).

9. Bar-Peled, L. et al. A tumor suppressor complex with GAP activity for the Rag GTPases that signal amino acid sufficiency to mTORC1. Science. 340 (6136), 1100-1106 (2013)

10. Ishida, S. et al. Mutations of DEPDC5 cause autosomal dominant focal epilepsies. Nature Genetics. (2013).

11. Dibbens, L.M. et al. Mutations in DEPDC5 cause familial focal epilepsy with variable foci. Nature Genetics. (2013).

12. Baulac, S., Weckhuysen, S. DEPDC5-Related Epilepsy. GeneReviews®. [bookaccession]. (1993).
13. Picard, F. et al. DEPDC5 mutations in families presenting as autosomal dominant nocturnal frontal lobe epilepsy. Neurology. (2014).

14. Teng, Y. et al. Knockdown of zebrafish Igi1a results in abnormal development, brain defects and a seizurelike behavioral phenotype. Human Molecular Genetics. (2010).

15. Baraban, S.C., Dinday, M.T., Hortopan, G.A. Drug screening in Scn1a zebrafish mutant identifies clemizole as a potential Dravet syndrome treatment. Nature Communications. (2013).

16. Suls, A. et al. De novo loss-of-function mutations in CHD2 cause a fever-sensitive myoclonic epileptic encephalopathy sharing features with dravet syndrome. American Journal of Human Genetics. (2013).

17. Grone, B.P. et al. Epilepsy, behavioral abnormalities, and physiological comorbidities in syntaxin-binding protein 1 (STXBP1) mutant zebrafish. PLoS ONE. (2016).

18. de Calbiac, H. et al. DEPDC5 knockdown causes mTORdependent motor hyperactivity in zebrafish. Annals of Clinical and Translational Neurology. (2018).

19. Panchaud, N., Péli-Gulli, M.P., De Virgilio, C. Amino acid deprivation inhibits TORC1 through a GTPase-activating protein complex for the Rag family GTPase Gtr1. Science Signaling. (2013).

20. Lim, K.R.Q., Maruyama, R., Yokota, T. Eteplirsen in the treatment of Duchenne muscular dystrophy. Drug Design, Development and Therapy. (2017).

21. Kok, F.O. et al. Reverse genetic screening reveals poor correlation between morpholino-induced and mutant phenotypes in zebrafish. Developmental Cell. (2015). 
22. Rossi, A. et al. Genetic compensation induced by deleterious mutations but not gene knockdowns. Nature. (2015).

23. Stainier, D.Y.R. et al. Guidelines for morpholino use in zebrafish. PLoS Genetics. (2017).

24. Kimmel, C.B., Ballard, W.W., Kimmel, S.R., Ullmann, B., Schilling, T.F. Stages of embryonic development of the zebrafish. Developmental dynamics: an official public. 203 (3), 253-310 (1995).

25. Møller, R.S., Dahl, H.A., Helbig, I. The contribution of next generation sequencing to epilepsy genetics. Expert Review of Molecular Diagnostics. (2015).

26. Allen, A.S. et al. De novo mutations in epileptic encephalopathies. Nature. (2013).

27. Dunn, P. et al. Next generation sequencing methods for diagnosis of epilepsy syndromes. Frontiers in Genetics. (2018).

28. Grone, B.P., Baraban, S.C. Animal models in epilepsy research: Legacies and new directions. Nature Neuroscience. (2015).

29. Zhang, Y. et al. Pharmacological characterization of an antisense knockdown zebrafish model of Dravet syndrome: Inhibition of epileptic seizures by the serotonin agonist fenfluramine. PLOS ONE. (2015).

30. Swaminathan, A. et al. Non-canonical mTORIndependent Role of DEPDC5 in Regulating GABAergic Network Development. Current Biology. (2018).

31. Samarut, É. et al. Y-Aminobutyric acid receptor alpha 1 subunit loss of function causes genetic generalized epilepsy by impairing inhibitory network neurodevelopment. Epilepsia. (September), 2061-2074 (2018).
32. Turrini, L. et al. Optical mapping of neuronal activity during seizures in zebrafish. Scientific Reports. (2017).

33. Rosch, R.E., Hunter, P.R., Baldeweg, T., Friston, K.J., Meyer, M.P. Calcium imaging and dynamic causal modelling reveal brain-wide changes in effective connectivity and synaptic dynamics during epileptic seizures. PLoS Computational Biology. (2018).

34. Baraban, S.C. Forebrain Electrophysiological Recording in Larval Zebrafish. Journal of Visualized Experiments. (2013).

35. Afrikanova, T. et al. Validation of the Zebrafish Pentylenetetrazol Seizure Model: Locomotor versus Electrographic Responses to Antiepileptic Drugs. PLoS ONE. (2013). 